Ann. Sci. forest., 1979, 36 (1), 13-38.

\title{
Etude du comportement du feu dans la Garrigue de Chêne kermes à partir des températures et des vitesses de propagation
}

\author{
L. TRABAUD \\ Département d'Ecologie générale \\ C.E.P.E. Louis-Emberger \\ Route de Mende, BP 5051, 34033 Montpellier Cedex
}

\begin{abstract}
Résumé
Le comportement d'un feu de végétation peut être caractérisé de diverses manières. Les températures, les vitesses de propagation, la hauteur des flammes, et l'énergie dégagée par le feu peuvent en être une représentation. Les températures varient en de rapides fluctuations; en outre, elles sont différentes selon l'emplacement des capteurs (dans le sol, dans la végétation, au-dessus de la végétation) et selon les époques de brûlages (printemps, automne). Les températures maximales les plus élevées sont toujours obtenues près du toit de la végétation et lors des brôlages d'automne. Compte tenu des résultats, un essai est réalisé pour présenter la structure de la flamme et la dispersion des températures dans le front de flammes. Les vitesses de propagation dépendent étroitement de la vitesse du vent et de la hauteur de la végétation. La hauteur de la flamme est fonction de la vitesse de propagation du feu et de la hauteur de la végétation. L'énergie dégagée par les feux est mesurée par la ". puissance linéaire du front du feu \%. Les énergies observées au cours des brûlages sont peu élevées.

Les résultats obtenus permettent de mieux comprendre le comportement du feu dans les garrigues de Chêne kermès. Ils présentent aussi une grande importance pour les opérations de lutte anti incendie.
\end{abstract}

\section{1. - Introduction}

L'évolution de la végétation méditerranéenne est souvent perturbée par le passage du feu. En France, c'est dans une région qui s'étend des Pyrénées-Orientales aux Alpes-Maritimes et à la Corse que les feux de végétation sont les plus nombreux et les plus dévastateurs. Chaque année, environ 30000 ha de végétation sont brûlés par les incendies.

Pour comprendre la dynamique de la végétation après incendie et connaître le « comportement du feu », nous avons effectué une série de brûlages sur des placettes situées dans une garrigue de Chêne kermès (Quercus coccifera L.). Le choix de cette communauté végétale est motivé par l'importance de cette unité parmi les peuplements végétaux du midi de la France où elle occupe plus de 100000 ha, et du fait aussi de la fréquence des feux qui la parcourent.

L'expression «comportement du feu » est utilisée pour désigner les divers phénomènes qui apparaissent au cours d'un feu (Barrows, 1951 ; Countryman, 1964 ; Mc Arthur et Cheney, 1970). Cette expression a, nécessairement, une large signifi- 
cation parce qu'un feu peut changer d'aspects de nombreuses fois au cours de son existence. En outre, ce « comportement» est si changeant que toute tentative pour le décrire ou le mesurer doit englober de nombreux paramètres. Les températures, les vitesses de propagation, les hauteurs des flammes en sont de bons exemples.

\section{1. - Sifuation de la station où ont été effectuées les recherches}

L'expérimentation pour étudier le comportement du feu dans la garrigue de Chêne kermès a été mise en place sur une colline au lieu-dit Puech-du-Mas-du-Juge (commune de St Gély-du-Fesc), à $10 \mathrm{~km}$ au nord de Montpellier. Cette colline a déjà fait l'objet de nombreuses études antérieures : Long et al. (1961, 1967), Trabaud (1962), Poissonet (1966).

Les conditions générales ont déjà été décrites (Trabaud, 1974, 1977). Les parcelles expérimentales sont situées sur le versant Sud-ouest de la colline ; la pente générale du terrain assez régulière, est comprise entre 10 ef 15 p. 100 ; l'altitude varie de 130 d $150 \mathrm{~m}$.

Le climat de la zone est méditerranéen humide selon la classification d'Emberger $(1942,1971)$ : le coefficient pluviothermique $Q_{2}$ est égal à 154,8 . La saison pluvieuse coīncide avec la période à faibles températures ; l'été est généralement chaud et sec. La moyenne annuelle des températures, à Saint-Gély-du-Fesc, est $14,4^{\circ} \mathrm{C}$; $\mathrm{m}$. la moyenne des températures minimales du mois le plus froid (janvier) est égal à, $2,4^{\circ} \mathrm{C} ; \mathrm{M}$. la moyenne des températures maximales du mois le plus chaud, est de $27,5^{\circ} \mathrm{C}$; les précipitations moyennes annuelles atteignent $1116,6 \mathrm{~mm}$.

Actuellement, la végétation dominante est une garrigue typique de Chêne kermès (Quercus coccifera L.) assez basse (entre 0,50 à $1 \mathrm{~m}$ de hauteur), de plus de 20 ans d'âge après le dernier feu sauvage.

\section{2. - Les méthodes et techniques utilisées}

\section{1. - Techniques utilisées pour l'enregistrement des fempératures}

Les propriétés requises pour qu'un appareillage puisse donner de bons résultats afin de connaître précisément les températures atteintes au cours des feux de végétation sont les suivantes :

- il faut qu'un large éventail de fempératures soit couvert ;

- l'appareillage doit être facilement transportable et adapté aux dures conditions de terrain :

- ses réactions doivent être rapides et sûres.

Les techniques qui répondent à ces exigences sont peu nombreuses. Les unes sont basées sur des instruments électriques, les autres sur des substances chimiques qui possèdent un point de fusion bien connu et dont l'état physique ne se modifie plus audelà de ce point. Dans les feux que nous avons allumés expérimentalement nous avons utilisé les deux techniques de mesure.

Les températures et leurs durées étaient enregistrées par des enregistreurs poten- 
tiométriques d̀ compensation de soudure froide à six voies chacun. Les vitesses d'enregistrement, comme les cadences, étaient variables et donc pouvaient être réglées en fonction des besoins de chaque expérience.

Deux séries de six thermocouples en nickel-chrome-nickel allié (chromel-alumel) étaient disposées à l'intérieur de la parcelle devant être brôlée. Chaque série était sifuée à un endroit différent de la parcelle. Les six thermocouples étaient placés au même emplacement à différentes hauteurs au-dessus du sol et dans le sol, de façon suivante:

- un thermocouple d̀ $1,50 \mathrm{~m}$ :

- un thermocouple à $1 \mathrm{~m}$;

- un hermocouple à $0,50 \mathrm{~m}$;

- un thermocouple à la surface du sol :

- deux thermocouples dans le sol, à deux profondeurs, dont un au niveau des racines, des rhizomes et des tiges souterraines, soit :

$$
\begin{aligned}
& \text { un à }-2,5 \mathrm{~cm} \text {, } \\
& \text { un d̀ }-5 \mathrm{~cm} \text {. }
\end{aligned}
$$

Au cours de plusieurs essais, nous avons installé des thermocouples à $10 \mathrm{~cm}$ de profondeur ; nous n'avons jamais observé des varictions de la température pendant ou après le feu.

Les fils des thermocouples étaient isolés par un guipage de soie de verre et d'amiante, l'ensemble recouvert par une tresse de soie de verre imprégnée aux silicones. Les portions des fils qui devaient traverser soit la végétation, soit le sol, étaient recouvertes par des isolateurs en «stéatite» spéciale peu conductrice de la chaleur. Enfin le tout était glissé dans des gaines de protection en céramique réfractaire ; seule la soudure du thermocouple dépassait du tube. Cela afin d'isoler parfaitement les fils des thermocouples pour éviter tout réchauffement parasite, ou pour éliminer au maximum les incidents électriques engendrant des impulsions parasites qui risquaient de modifier les données enregistrées.

Pour faire parvenir les fils des thermocouples aux enregistreurs, et afin de ne pas endommager la végétation en place, les fils étaient, ensuite, glissés dans de longs tubes de métal qui étaient disposés à même le sol entre les souches.

Par ailleurs, des bandes verticales de vernis (tabl. 1) dort les points de fusion correspondent à des températures connues (Fenner et Bentley, 1960), étaient peintes sur des plaquettes de mica $(5 \times 10 \mathrm{~cm})$. Ensuite, la plaquette de mica, côté peint, était placée sur une plaquette d'amiante de façon à éliminer des dégâts possibles causés

\section{TABLEAU 1}

Températures de virage des vernis utilisés au cours des feux expérimenfaux ( $\left.{ }^{\circ} \mathrm{C}\right)$

\begin{tabular}{|c|c|c|c|c|c|c|c|c|c|}
\hline $149^{\circ}$ & $198^{\circ}$ & $253^{\circ}$ & $316^{\circ}$ & $399^{\circ}$ & $500^{\circ}$ & $593^{\circ}$ & $704^{\circ}$ & $804^{\prime \prime}$ & $899^{\circ}$ \\
\hline \multicolumn{10}{|c|}{ Températures de virage des vernis utilisés dans le sol } \\
\hline 390 & $48^{\circ}$ & $55^{\circ}$ & $59^{\circ}$ & $69^{\circ}$ & $79^{\circ}$ & $90^{\circ}$ & $98^{\circ}$ & $104^{\circ}$ & $149^{\circ}$ \\
\hline
\end{tabular}

(Critical temperatures of the thermal indicators used in the experimental fires ( ${ }^{\circ} \mathrm{C}$ ) 
aux bandes par les manipulations ou la déposition des produits de combustion. L'ensemble permettant cependant le libre passage de la chaleur. Le tout était attaché avec du fil de cuivre. Le mica et l'amiante étaient utilisés car, en plus d'être incombustibles, ce sont de très faibles conducteurs de la chaleur. Les plaquettes ainsi réalisées peuvent être suspendues à des piquets en fer ou enfoncées dans le sol. Chaque vernis change de couleur et de structure à une température donné : la couleur pâlit et la peinture cristallise quand la température critique de virage est atteinte. Cette différence d'état est clairement marquée et irréversible.

Les deux techniques d'enregistrement ont été utilisées car elles apportent des données complémentaires quant à la durée et la distribution des températures dans l'espace et dans le temps.

\section{2. - Techniques d'étude utilisées pour observer et analyser les vitesses de propagation}

Pour observer les vitesses de propagation, nous avons placé des piquets de repérage tous les $2 \mathrm{~m}$ tout autour des parcelles. Ensuite pendant le feu sur un plan de la parcelle, un chronométreur notaif l'emplacement du front de flammes en train de progresser. La vitesse moyenne de propagation correspond alors au nombre de mètres parcourus par le feu divisé par le temps mis par le front de flammes pour traverser la longueur totale de la parcelle; nous obtenons ainsi la vitesse atteinte par la tête du front de flammes.

\section{3. - Techniques de mises à feu, bordereaux d'enregistrement des données et observations des facteurs atmosphériques}

Tous les feux ont été allumés au moyen de lance-flammes agricoles afin de réaliser un front de flammes continu et semblable à celui d'un feu qui a atteint son stade d'équilibre et se propage librement. Toutes les observations que nous avons faites et qui constituent la base de cet article proviennent de zones où le feu brôlait activement et se propageait naturellement.

Les feux dits de « printemps » ont été allumés à la fin mai début juin, ceux d'« automne» foujours dans la première quinzaine de septembre.

A l'origine (garrigue de 20 ans environ après le dernier feu sauvage) la hauteur de la végétation atteignait $1 \mathrm{~m}$ à $1,50 \mathrm{~m}$. Par la suite, des mesures ont été réalisées sur des parcelles déjà brôlées et dont la végétation était moins élevée.

Les parcelles avaient toutes une superficie de $50 \mathrm{~m}^{2}(5 \times 10 \mathrm{~m})$. Cependant au cours de deux expériences supplémentaires, nous avons brôlé deux parcelles de $200 \mathrm{~m}^{2}(10 \times 20 \mathrm{~m})$ chacune.

Les données concernant les conditions météorologiques du moment, ainsi que l'état de la végétation étaient notés sur un bordereau spécial d'observation. En effet, les conditions météorologiques et les caractéristiques structurales de la végétation ont une action sur le feu et influent sur son comportement. Les données correspondant à la température et à l'humidité relative de l'air proviennent d'un poste météoroloqique classique implanté au milieu de la zone expérimentale.

Ełant donné que le vent influence la propagation du feu, sa vitesse est mesurée par un anémomètre placé à deux mètres au-dessus du sol. La direction est donnée 
par un drapeau. Si nous avons placé l'anémomètre si bas, soit à peu près à la hauteur de la végétation, c'est que le vent est généralement mesuré dans les stations météorologiques classiques à $10 \mathrm{~m}$ environ au-dessus du sol; or, le vent qui influence la propagation du feu est celui qui atteint le front de flammes, donc celui qui se déplace au-dessus et dans la couche combustible.

Enfin l'état de la végétation est extrémement important, particulièrement sa teneur en eau qui détermine son degré de furgescence et la vitesse de propagation du feu ; mais aussi sa densité, ou l'inverse, sa porosité. Les graminées jouent aussi un rôle capital car ce sont elles qui transmettent le plus rapidement la flamme quand elles sont sèches, ou, au contraire, si elles sont gorgées d'eau ralentiront notablement la vitesse de propagation (Trabaud, 1976).

La composition floristique a aussi son importance ; selon la proportion des différentes espèces, le peuplement sera plus ou moins inflammable ef combustible.

\section{3. - Les résultats}

\section{1. - Les températures enregistrées au cours des feux}

\subsection{Revue de la documentation existante.}

Les températures atteintes lors d'un feu sont un facteur important du comportement du feu, en relation avec l'époque du feu et la phytomasse des espèces dominantes.

Jusqu'à ce jour, toutes les éfudes entreprises sur la connaissance des températures au cours de feux sauvages ou de feux contrôlés ont été réalisées dans des pays autres que la France et, par conséquent, dans des végétations totalement différentes de celles des garrigues méditerranéennes.

Whittaker (1961) brôlant des landes de Callune en Ecosse (Grande-Bretagne) trouve des températures de l'ordre de 600 à $840 \circ$, à $20 \mathrm{~cm}$ de hauteur dans la touffe de Callune. Kayll (1966) dans le même type de végétation enregistre des températures maximales de $500^{\circ} \mathrm{C}$ environ, pour les feux d'automne, alors que les feux de printemps, moins intenses, ne dépassent pas $350^{\circ} \mathrm{C}$.

Dans le Nord de l'Ontario (Canada), avec une végétation de Graminées mêlées à des buissons bas, Smith et Sparling (1966) décrivent trois types de feux : des feux « peu intenses » au cours desquels la température maximale est toujours inférieure à $300^{\circ} \mathrm{C}$; des feux «moyens» dont les températures sont comprises entre $200^{\circ}$ et $400^{\circ} \mathrm{C}$; enfin des feux « intenses » dont la température maximale dépassait toujours $400^{\circ} \mathrm{C}$. La température maximale était enregistrée, habituellement, entre 5 et $10 \mathrm{~cm}$ audessus du sol. Les feux « les plus intenses » ayant leurs températures maximales à des hauteurs plus élevées que celles des feux «peu intenses».

Stinson et Wright (1969) au Texas (Etats-Unis) enregistrent, à la surface du sol, des températures variant, en moyenne, entre $280^{\circ}$ et $390^{\circ} \mathrm{C}$ dans des feux parcourant des peuplements graminéens.

Au Sénégal, Pitot et Masson (1951), en brôlant une savane obtiennent des températures de $90^{\circ}$ à $140^{\circ} \mathrm{C}$, à des thermocouples placés au niveau du sol ; à $5 \mathrm{~cm}$ de 
hauteur, la température oscille entre $285^{\circ}$ et $560^{\circ} \mathrm{C}$ et à $1,40 \mathrm{~m}$, les températures allaient de $140^{\circ}$ à $375^{\circ} \mathrm{C}$. Aucune température supérieure à $200^{\circ} \mathrm{C}$ ne persistait audelà d'une minute. A la surface du sol, les températures supérieures à $50^{\circ} \mathrm{C}$ duraient pendant 1 à $3 \mathrm{mn}$.

Enfin, au Japon, Iwanami (1973), dans une série d'études sur les températures des feux de peuplements graminéens, montre que plus la phytomasse des peuplements est élevée, plus les températures sont fortes. La valeur des températures oscillait entre $350^{\circ}$ et $880^{\circ} \mathrm{C}$. Les températures les plus élevées étaient toujours observées entre 5 et $25 \mathrm{~cm}$ au-dessus du sol.

Apparemment, les températures dépendent du type de combustible de sa taille de sa distributon spatiale et de sa teneur en eau, ainsi que de la vitesse du vent. En effet. Whittaker (1961) observe qu'un accroissement de la vitesse du vent entraine une augmentation de la température enregistrée de l'ordre de $77^{\circ}$ à $155^{\circ} \mathrm{C}$.

II faut ajouter que ces auteurs ont travaillé dans des types de végétation plus ouverts, ou ayant une plus grande proportion de Graminées, de moindre hauteur que ceux de la région méditerranéenne française, ce qui fait que leurs valeurs peuvent paraître assez faibles. Cependant, Martin et al. (1969), en Géorgie (Etats-Unis), lors de feux «prescrits», enregistrent des températures de $700^{\circ} \mathrm{C}$ à $30 \mathrm{~cm}$ au-dessus du sol, et de $500^{\circ} \mathrm{C}$ à $1,70 \mathrm{~m}$. Ces auteurs constatent que la température est plus élevée quand la vitesse de propagation du feu est plus rapide.

\subsection{Les courbes d'enregistrement.}

Tous les enregistrements des températures effectués lors des feux expérimentaux présentent des courbes qui ont la même allure. Le front de flamme arrive, passe au niveau de l'emplacement du thermocouple, il y a alors élévation de la température. puis dépasse cet emplacement et continue sa course; les températures décroissent ensuite plus ou moins rapidement.

Selon la position du thermocouple dans l'espace (dans la végétation, au niveau du sol ou dans le sol), les courbes d'enregistrement ont des allures légèrement différentes et les températures maximales atteintes sont différentes.

En l'espace de quelques secondes, les températures varient en de rapides fluctuations de grande amplitude. Ceci à cause des oscillations convulsives des flammes. Cependant, plus la surface du sol est proche, plus les fluctuations des températures s'atténuent ef l'amplitude thermique diminue.

Parmi les soixante six enregistrements des températures obtenus au cours des brûlages, et du fait de leur grande similitude, nous n'examinerons que trois cas bien précis et représentatifs.

\subsection{Feu de printemps, premier brûlage (fig. 1).}

\section{a) Conditions du feu.}

Beau temps ensoleillé ; vent de WNW : $2,5 \mathrm{~m} \cdot \mathrm{s}^{-1}$; température de l'air : $22{ }^{\circ} \mathrm{C}$; humidité relative de l'air : 23 p. 100 ; teneur en eau des végétaux : 48,5 p. 100 ; hauteur de la végétation : $1 \mathrm{~m}$; phytomasse aérienne estimée : $20 \mathrm{t}$. ha $\mathrm{a}^{-1}$; vitesse de propagation du feu : $0,04 \mathrm{~m}+\mathrm{s}^{-1}$. 


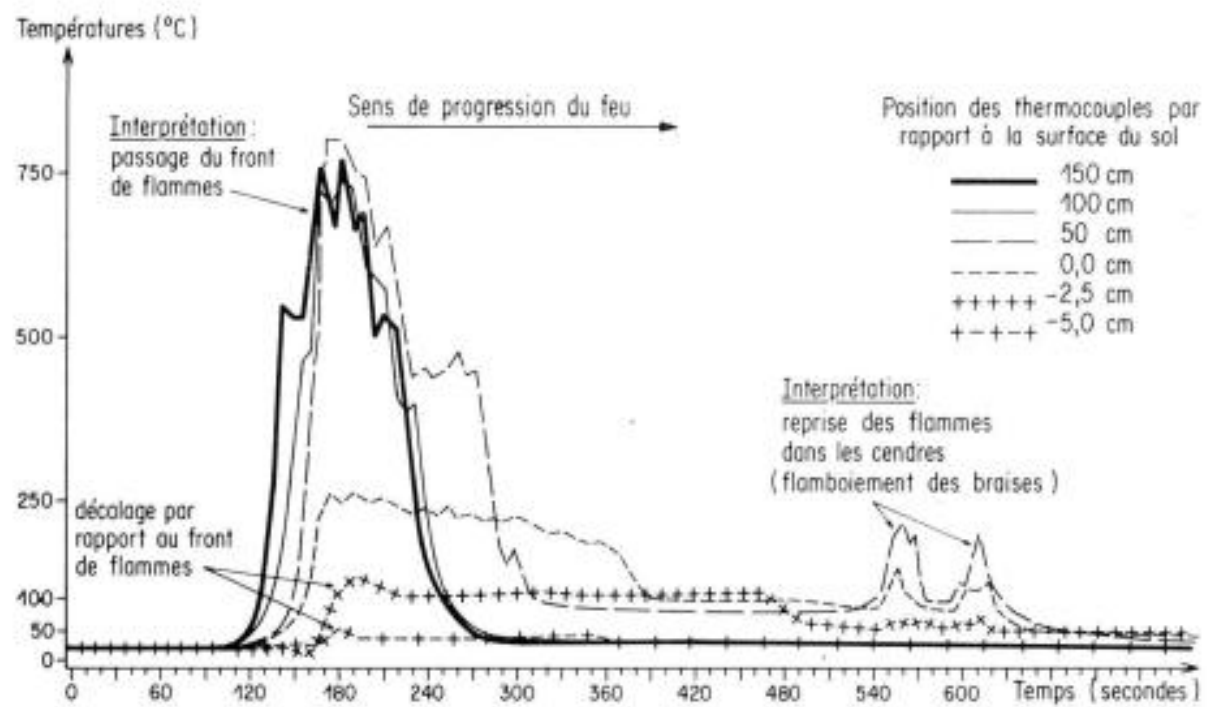

FIG. 1. - Courbes d'enregistrement des tempérafures lors d'un feu de « printemps ». végétation initiale : $1 \mathrm{~m}$ de hauteur, $20 \mathrm{tde}$ MS. ha- $\mathrm{a}^{-1}$; vent : $2,5 \mathrm{~m} \cdot \mathrm{s}^{-1}$; hauteur du front de flammes : $1,80 \mathrm{~m}$.

Temperature recorded curves during a \& spring * fire. Initial vegetation : $1 \mathrm{~m}$ high, $20 \mathrm{DMt}, \mathrm{ha}^{-1}$; wind speed : $2.5 \mathrm{~m} \cdot \mathrm{s}^{-1}$; flame front height $: 1.80 \mathrm{~m}$.

\section{b) Températures au-dessus du sol.}

Avant le passage du front de flammes, il n'y a aucune élévation de la température de l'air jusqu'à ce que le front soit assez proche des thermocouples. C'est le thermocouple situé à $1,50 \mathrm{~m}$ de hauteur qui le premier décèle une élévation de la température (jusqu'à $550^{\circ} \mathrm{C}$ ).

Au passage du front de flammes, tous les thermocouples enregistrent l'élévation (jusqu'à $750^{\circ} \mathrm{C}$ ). Les thermocouples situés au-dessus de la végétation présentent des températures légèrement inférieures au thermocouple placé dans la végétation $(0,50 \mathrm{~m})$; celui-ci, pendant une dizaine de secondes, enregistre $800^{\circ} \mathrm{C}$; mais du fait de sa position, n'enregistre pas les oscillations des températures. Par contre, les deux autres thermocouples situés hors de la végétation montrent des écarts de l'ordre de $100^{\circ} \mathrm{C}$.

Le passage de la tête du feu ne dure environ qu'une minute ; de ce fait, les thermocouples placés hors de la végétation n'enregistrent la température que pendant ce laps de temps. Par contre, le thermocouple placé dans la végétation continue à enregistrer des températures élevées ; cela est dû à l'épaisseur de la zone en flamme dans la végétation.

c) Températures au niveau du sol.

L'élévation de la température apparaît plus tardivement que celle enregistrée au-dessus du sol. En outre, la température atteinte $\left(25^{\circ} \mathrm{C}\right)$ est très inférieure à celles atteintes au-dessus du sol. Cependant, cette température, du fait des braises chaudes, puis des cendres, va persister longuement $(3 \mathrm{mn})$ avant de descendre à $100^{\circ} \mathrm{C}$. La 
courbe présente une série décroissante de petits pics en dents de scie, mettant en évidence de petites pulsations thermiques à l'intérieur des cendres.

d) Températures dans le sol.

Tout d'abord, il faut noter le décalage important, dix secondes environ, entre le début du passage du front de flammes et l'élévation de température.

A moins $2,5 \mathrm{~cm}$, la température s'élève jusqu'à environ $125^{\circ} \mathrm{C}$, puis s'abaisse un peu aux alentours de $100^{\circ} \mathrm{C}$ et persiste pendant très longtemps : environ $15 \mathrm{mn}$ avant de revenir à la température ambiante. A ce niveau-ci, il faut noter un abaissement de la température (de l'ordre de $10^{\circ} \mathrm{C}$ ) au moment du passage de la tête du front, mettant en évidence la présence d'une circulation d'air frais au niveau des premiers centimètres du sol.

A moins $5 \mathrm{~cm}$, la température s'élève très légèrement, jusqu'à $50^{\circ} \mathrm{C}$, puis diminue régulièrement pour revenir à celle d'origine.

e) Températures au cours de la période de refroidissement.

Quelques courbes de la figure 1 présentent une brusque légère élévation de la température, cela est dô à la reprise des flammes dans les cendres (flamboiement des braises).

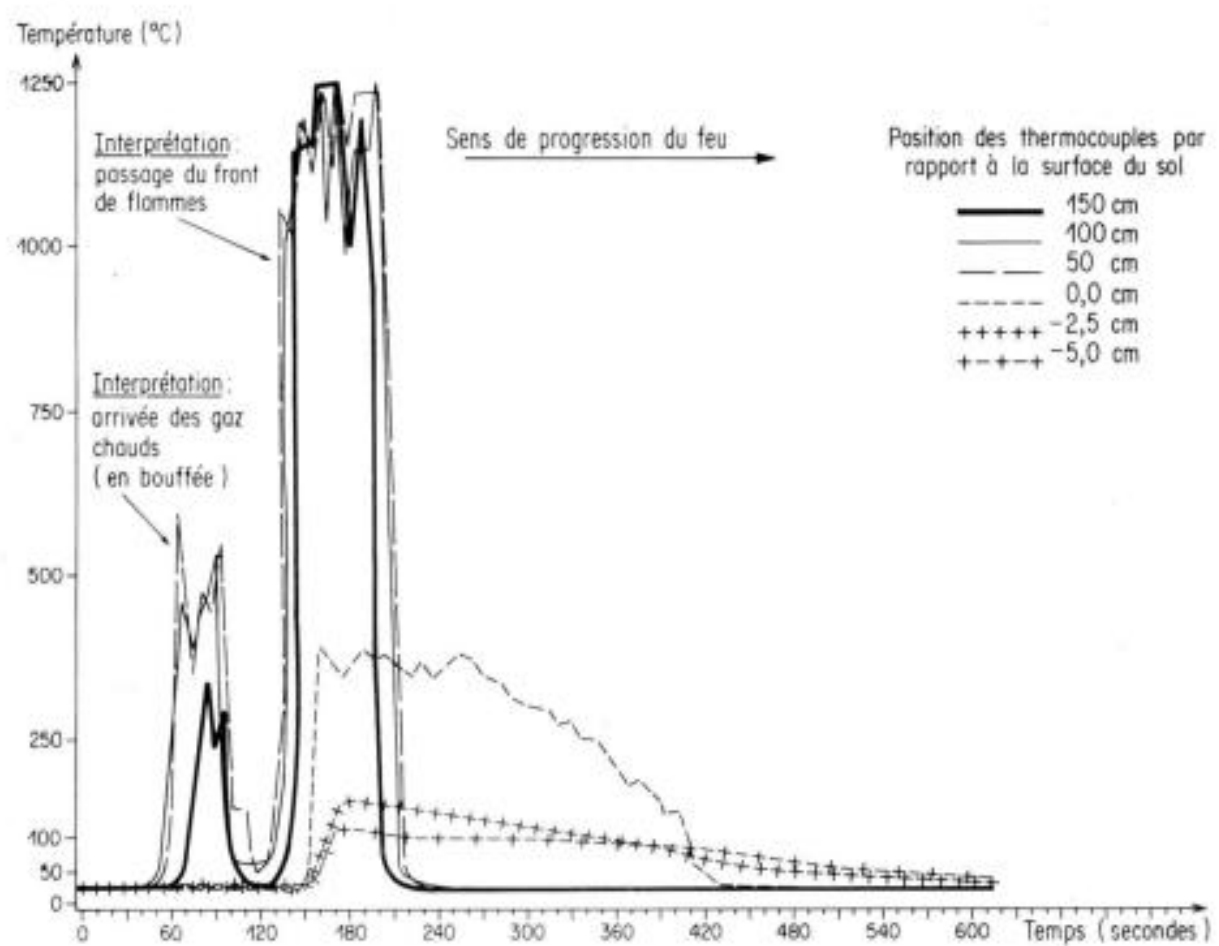

FIG. 2. - Courbes d'enregistrement des température lors d'un feu $d^{\prime} \alpha$ automne ». Végétation initiale : $0,80 \mathrm{~m}$ de hauteur, $18+$ de MS. ha $a^{-1}$; vent : $3 \mathrm{~m} \cdot \mathrm{s}^{-1}$; hauteur du front de flammes: $1,80 \mathrm{~m}$.

Temperature recorded curves during an o autumn $x$ fire. Initial vegetation :0.80 m high, $18 \mathrm{DMt}$. hawind speed : $3 \mathrm{~m} \cdot \mathrm{s}^{-1}$, flame front height $: 1.80 \mathrm{~m}$. 


\subsection{Feu d'automne, premier brûlage (fig. 2).}

a) Conditions du feu.

Temps nuageux clair (50 p. 100 cirro-stratus) ; vent NW, irrégulier : $3 \mathrm{~m} \cdot \mathrm{s}^{-1}$; température de l'air : $3 \mathrm{~m} . \mathrm{s}^{-1}$; température de l'air : $22^{\circ} \mathrm{C}$; humidité relative de l'air : 30 p. 100 ; teneur en eau des végétaux : 54,4 p. 100 ; hauteur de la végétation : $0,80 \mathrm{~m}$; phytomasse aérienne estimée : 18 †. ha- ${ }^{-1}$; vitesse de propagation du feu : $0,037 \mathrm{~m} \cdot \mathrm{s}^{-1}$.

A première vue, les courbes d'enregistrement paraissent assez différentes de celles du feu de printemps. Ce n'est qu'une apparence due aux conditions météorologiques, en particulier la vitesse du vent.

b) Températures au-dessus du sol.

Les températures sont plus élevées que pour le feu de printemps quel que soit l'emplacement des thermocouples.

Un peu avant le passage du front de flammes, les thermocouples placés au-dessus du sol, et plus particulièrement ceux situés à $0,50 \mathrm{~m}$ et $1 \mathrm{~m}$, enregistrent une élévation de la température de l'ordre de $500^{\circ} \mathrm{C}$ environ due à une arrivée des gaz chauds en bouffées poussées par le vent. La rafale du vent tombant, les bouffées d'air chaud cessent quelque temps, puis arrive le front de flammes. L'élévation des températures est alors brutale : $1250^{\circ} \mathrm{C}$. Ces températures vont persister le temps du passage du front de flammes ( $1 \mathrm{mn}$ environ), puis vont «tomber» brutalement.

Les différences par rapport au feu de printemps résident dans les températures maximales atteintes : $1250^{\circ} \mathrm{C}$, au lieu de $800^{\circ} \mathrm{C}$, et la rapidité de passage du front du feu. Si toutes les températures maximales ne sont pas aussi élevées, ces températures de l'ordre de $1000^{\circ} \mathrm{C}$ caractérisent cependant les feux d'automne.

c) Tempérafures au niveau du sol.

Du fait des températures élevées, le thermocouple situé au niveau du sol enregistre une élévation de température $\left(400^{\circ} \mathrm{C}\right)$ supérieure à celle du printemps. Comme pour la courbe de température du printemps, celle d'automne présente une série de dents de scie, ainsi qu'un abaissement progressif de la température. Mais, dans ce cas, la température n'atieint $100^{\circ} \mathrm{C}$ qu'après un laps de temps plus long qu'au printemps : $4 \mathrm{mn}$.

d) Températures dans le sol.

Comme au printemps, l'élévation de la température apparaît avec un certain retard (environ $15 \mathrm{~s}$ après l'arrivée du front de flammes). Cependant, aussi bien à moins $2.5 \mathrm{~cm}$ qu'à mcins $5 \mathrm{~cm}$, cette élévation sera supérieure à celle du printemps : $150^{\circ} \mathrm{C}$ respectivement. L'abaissement de la température au passage de l'air existe toujours, quoique un peu moins marquée.

Très lentement, les températures tendent à revenir au niveau des températures avant le feu ; cependant, à l'inverse du printemps, le thermocouple placé à moins $5 \mathrm{~cm}$ montre une perte de chaleur moins rapide que le thermocouple placé à moins $2,5 \mathrm{~cm}$. Mauvaise conductrice de la chaleur, la terre s'échauffe lentement, mais, par contre, conserve plus longtemps la chaleur. 
3.123. Feu d'automne, végétation ayant subi une fréquence élevée de brûlages (troisième brûlage en 6 ans).

a) Conditions du feu.

Beau temps ensoleillé (moins de 10 p. 100 de cirro-stratus) ; vent ESE : 2 m.s $\mathrm{s}^{-1}$; température de l'air : $25^{\circ} \mathrm{C}$; humidité relative : 29 p. 100 ; teneur en eau des végétaux : 39,3 p. 100 ; hauteur de la végétation : $0,40 \mathrm{~m}$; phytomasse aérienne estimée : 6 t. ha ${ }^{-1}$; vitesse de propagation du feu : $0,025 \mathrm{~m} \cdot \mathrm{s}^{-1}$.

Le feu a été allumé dans une parcelle brôlée tous les deux ans en automne. La végétation de $40 \mathrm{~cm}$ de hauteur environ est composée principalement par Brachypodium ramosum.

b) Les températures.

Les courbes d'enregistrement (fig. 3) sont identiques à celles du premier brûlage au printemps. Les gaz chauds se font sentir un peu avant le passage du front de flammes. Celui-ci, poussé par le vent et courant dans une végétation à Graminées dominantes, n'est pas très profond : la durée de son passage est assez courte, $30 \mathrm{~s}$.

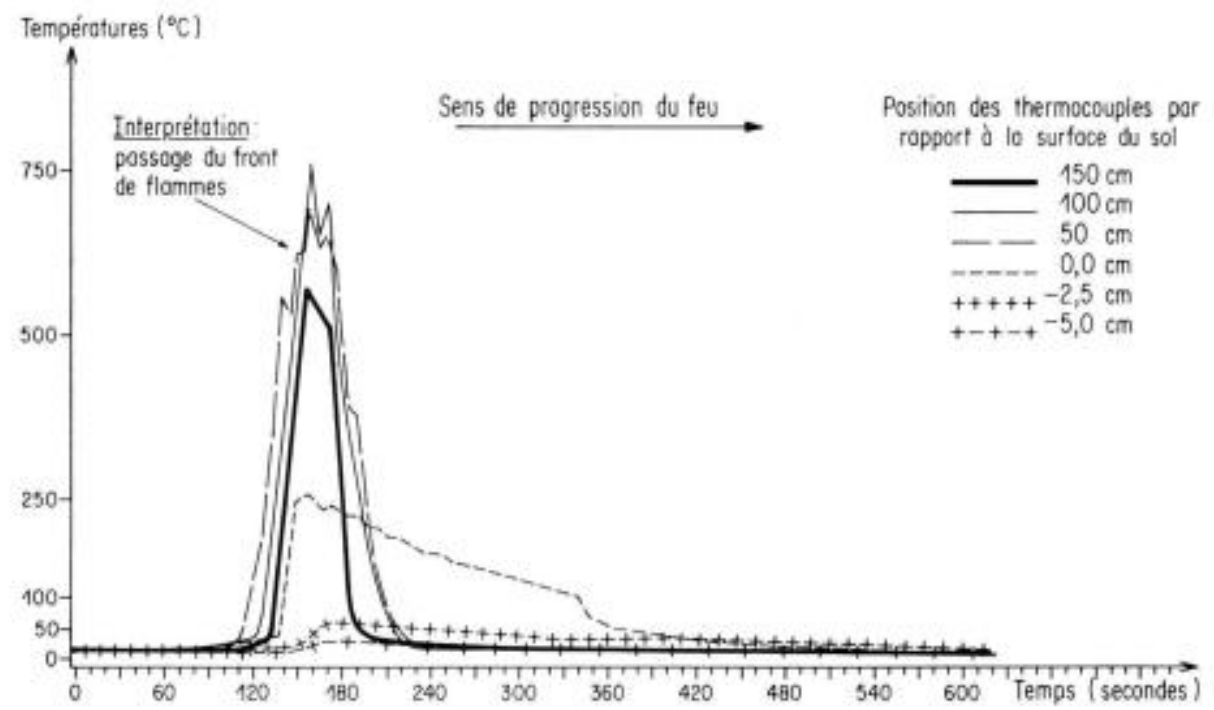

FIG. 3. - Courbes d'enregistrement des fempératures lors $d^{\prime}$ un feu $d^{*}$ \& automne $*$. Végétation :

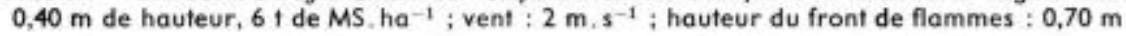

Temperature recorded curves during an \& autumn * fire. Vẻgétation : $0.40 \mathrm{~m}$ high, $6 \mathrm{DMt}, \mathrm{ha}^{-1}$; wind speed : $2 \mathrm{m.s^{-1 }}$; flame front height : $0.70 \mathrm{~m}$.

Les températures maximales enregistrées sont situées à des niveaux $(0,50$ et $0,25 \mathrm{~m}$ ) plus bas que précédemment ; ceci est dû à la hauteur de la végétation. A $1 \mathrm{~m}$, c'est-à-dire à $0,60 \mathrm{~cm}$ au-dessus du toit de la végétation, la température maximale n'atteint que $625^{\circ} \mathrm{C}$.

Au niveau du sol, la courbe de température est identique à celle du premier brûlage de printemps; la seule différence consiste en une diminution de la chaleur, 
beaucoup plus rapide, sans reprise de flammes, car les touffes de Graminées entretiennent mal la calcination, il n'y a donc pas de braises ni de cendres suffisamment chaudes pour conserver la chaleur ( $3 \mathrm{mn}$ pour atteindre $50^{\circ} \mathrm{C}$ ).

Dans le sol, l'accroissement de température est assez faible : $60^{\circ} \mathrm{C}$ pour moins $2,5 \mathrm{~cm}$ et $10^{\circ} \mathrm{C}$ de différence pour le thermocouple situé à moins $5 \mathrm{~cm}$.

\subsection{Les températures au-dessus du feu.}

Afin d'avoir une idée des températures à la base de la colonne de convection, nous avons brôlé deux parcelles de $200 \mathrm{~m}^{2}$ chacune. Seules les températures nettement au-dessus du sol (c'est-à-dire à partir de $3 \mathrm{~m}$ de hauteur) ont été enregistrées. Pour ce faire, un bâti, relativement léger, a été construit ; ce dispositif permet de placer les thermocouples à différentes hauteurs à partir du niveau du sol et donc, de connaître les températures afteintes à chaque niveau (tabl. 2).

\section{TABLEAU 2}

Températures enregistrées a la base de la colonne de convection dans une garrigue de Chêne Kermès (Recorded femperatures at the bottom of the convection column in a Quercus coccifera garrigue fire)

\begin{tabular}{ccc}
\hline $\begin{array}{c}\text { Hauteurs } \\
\text { au-dessus du sol } \\
(\mathrm{m})\end{array}$ & $\begin{array}{c}\text { Température moyenne } \\
\text { du front de flammes } \\
\text { en }{ }^{\circ} \mathrm{C}\end{array}$ & $\begin{array}{c}\text { Température maximale } \\
\text { du front de flammes } \\
\text { en }{ }^{\circ} \mathrm{C}\end{array}$ \\
\hline 6,50 & 55 & 62 \\
6,00 & 54 & 68 \\
5,00 & 67,5 & 87 \\
4,50 & 138 & 130 \\
4,00 & 139 & 175 \\
3,50 & 189 & 218 \\
\hline
\end{tabular}

Si ces températures paraissent relativement faibles par rapport à celles enregistrées dans la végétation, il faut dire que, du fait des difficultés rencontrées pour installer une telle expérimentation, les capteurs n'étaient pas placés rigoureusement au centre des parcelles, que l'enregistrement effectif du passage des flammes n'a duré que quelques minutes et que le vent, amenant sans cesse de l'air frais par dessus les flammes a pu jouer un rôle en établissant un « écran » entre les flammes et les thermocouples.

\subsection{Interprétation des résultats.}

Jusqu'à présent nous n'avons traité que des températures enregistrées par les thermocouples. Grâce au grand nombre de plaquettes portant des vernis, installées dans les parcelles lors des feux contrôlés, nous pouvons essayer de vérifier et compléter les résultats déjà obtenus à l'aide des thermocouples.

3.131. Températures observées au-dessus du sol.

a) Dans la végétation originelle.

Lors des premiers brôlages, la hauteur moyenne de la végétation atteint $1,00 \mathrm{~m}$. Tout d'abord, une première constation s'impose : les feux d'automne ont des tempé- 
ratures plus élevées que les feux de printemps (fig. 4). Ensuite, selon la hauteur à laquelle sont placées les plaquettes les indices thermiques enregistrés diffèrent.

A $1,50 \mathrm{~m}$, en automne, toutes les « températures 》 sont situées au-delà de $800^{\circ} \mathrm{C}$, et plus particulièrement entre 800 ef $850^{\circ} \mathrm{C}(80 \mathrm{p}$. 100) ; par contre, au printemps, cette tendance est presque l'inverse, puisque 77,5 p. 100 des « températures 》 atteintes lors des feux de cette période sont compris entre 0 et $175^{\circ} \mathrm{C}$.

A $1,00 \mathrm{~m}, 70$ p. 100 des feux d'automne ont des « températures » à $650^{\circ} \mathrm{C}$, alors que seulement 15 p. 100 des «températures» des feux de printemps atteignent ce niveau ou le dépassent.

A $0,50 \mathrm{~m}$, au milieu de la végétation, 82 p. 100 des « températures 》 automnales sont supérieures d̀ $650^{\circ} \mathrm{C}$. Même les «températures» de printemps dépassent ce seuil : 50 p. 100 des observations faites à ce niveau (fig. 4).

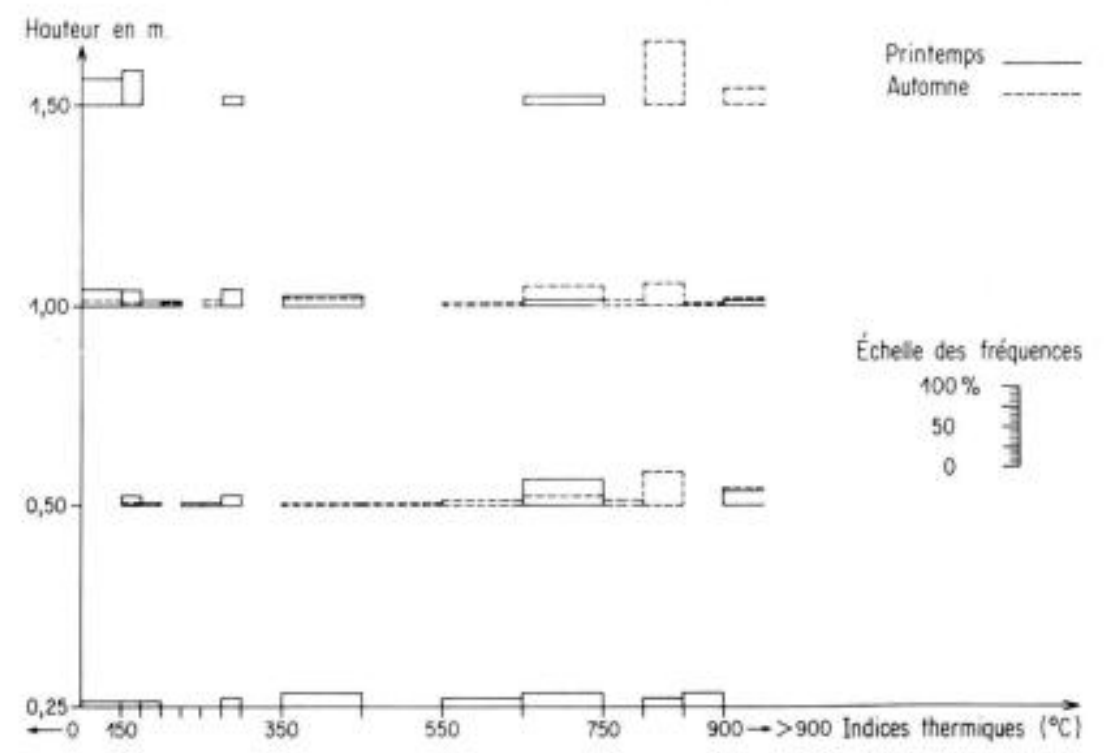

FIG. 4. - Fréquence (en p. 100) des indices thermiques enregistrés, en fonction de l'emplacement des indicateurs thermiques et de lo période de mise d̀ feu. Végétation originelle $(1-1,50 \mathrm{~m}$ de hauteur ; 18-20 i de MS, $\mathrm{ha}^{-1}$ ).

Frequency (in p. 100) of the recorded thermal indices, according to the location of the thermal indicators and the time of burning. Initial vegetation (1-1.50 m high : 18-20 $\mathrm{DMt}_{\text {. ha }}{ }^{-1}$ ).

Enfin, à $0,25 \mathrm{~m}$, très proche donc du niveau du sol, les « températures » se répartissent à peu près de façon équilibrée entre les fortes valeurs et les valeurs faibles : 45 p. 100 des «températures » dépassent $650^{\circ} \mathrm{C}$.

b) Dans la végétation déjà brûlée.

La hauteur moyenne de la végétation afteint $0,50 \mathrm{~m}$ environ.

Les «températures» observées sur les plaquettes sont plus faibles que celles observées lors des premiers feux (fig. 5). Mais ce sont toujours les feux d'automne qui présentent les «températures» les plus élevées. 
Lors de ces brûlages, rares sont les « températures 》 qui dépassent $650^{\circ} \mathrm{C}$. Elles sont toutes sifuées au niveau du toit de la végétation (66 p. 100) ou légèrement audessus, et ne sont enregistrées qu'au cours des feux d'automne.

Les « températures » des brûlages de printemps sont très peu élevées et, sauf au niveau $0,25 \mathrm{~m}$, ne dépassent jamais $550^{\circ} \mathrm{C}$ (fig. 5).

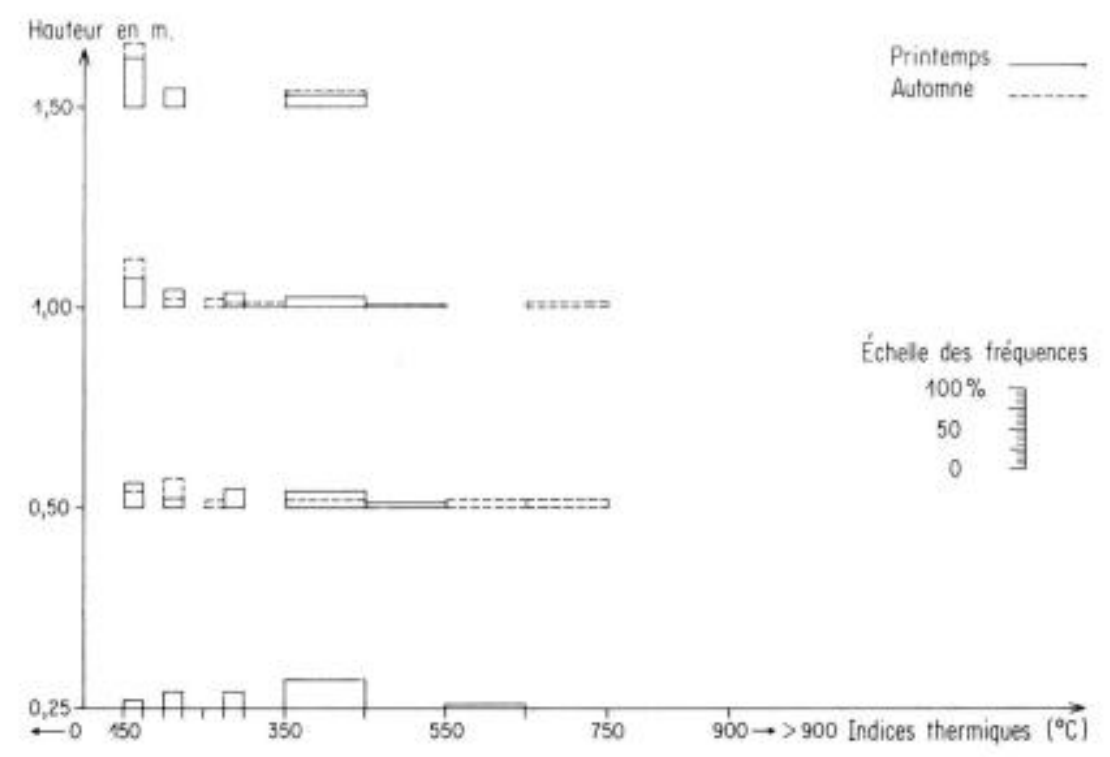

FIG. 5. - Fréquence (en p. 100) des indices thermiques enregistrés, en fonction de l'emplocement des indicateurs thermiques et de la période de mise d feu (végétation : $0,50 \mathrm{~m}$ de hauteur : $5-6 \mathrm{t}$ de MS. ha ${ }^{-1}$ ).

Frequency (inp. 100) of the recorded thermal indices, according to the location of the thermal indicators and the time of burning (vegetation : 0,50 high ; 5-6 DMt. ha ${ }^{-1}$ ).

c) Fréquence des «fempératures》 en fonction de la hauteur de la végétation.

Les températures les plus fortes sont observées au niveau du toit de la végétation ou légèrement au-dessus (fig. 6 ef 7). Plus l'indicateur thermique est placé haut et plus la végétation est basse, plus les « températures » enregistrées sont faibles. Par contre, au fur et à mesure que l'indicateur thermique est proche du toit de la végétation, les « températures» obtenues sont plus élevées. Cela est particulièrement net pour les niveaux $1,00 \mathrm{~m}$ et $0,50 \mathrm{~m}$ pour les feux de printemps (fig. 6 ) et $1,50 \mathrm{~m}$ et $1,00 \mathrm{~m}$ pour les feux d'automne (fig. 7).

Par contre, les indicateurs thermiques à $0,25 \mathrm{~m}$ pour les feux de printemps et ceux placés à $0,50 \mathrm{~m}$ pour les feux d'automne, donnent des indications moins nettes et plus diffuses ; en effet, dès que le toit de la végétation est situé au-dessus des plaquettes. les différences de «températures » correspondant aux différentes hauteurs de végétation sont moins nettes.

Les « températures » les plus élevées sont situées au niveau du toit de la végétation ou un peu au-dessus. Il y a une diminution de la «température » nettement au-dessus du toit de la végétation et en-dessous. 


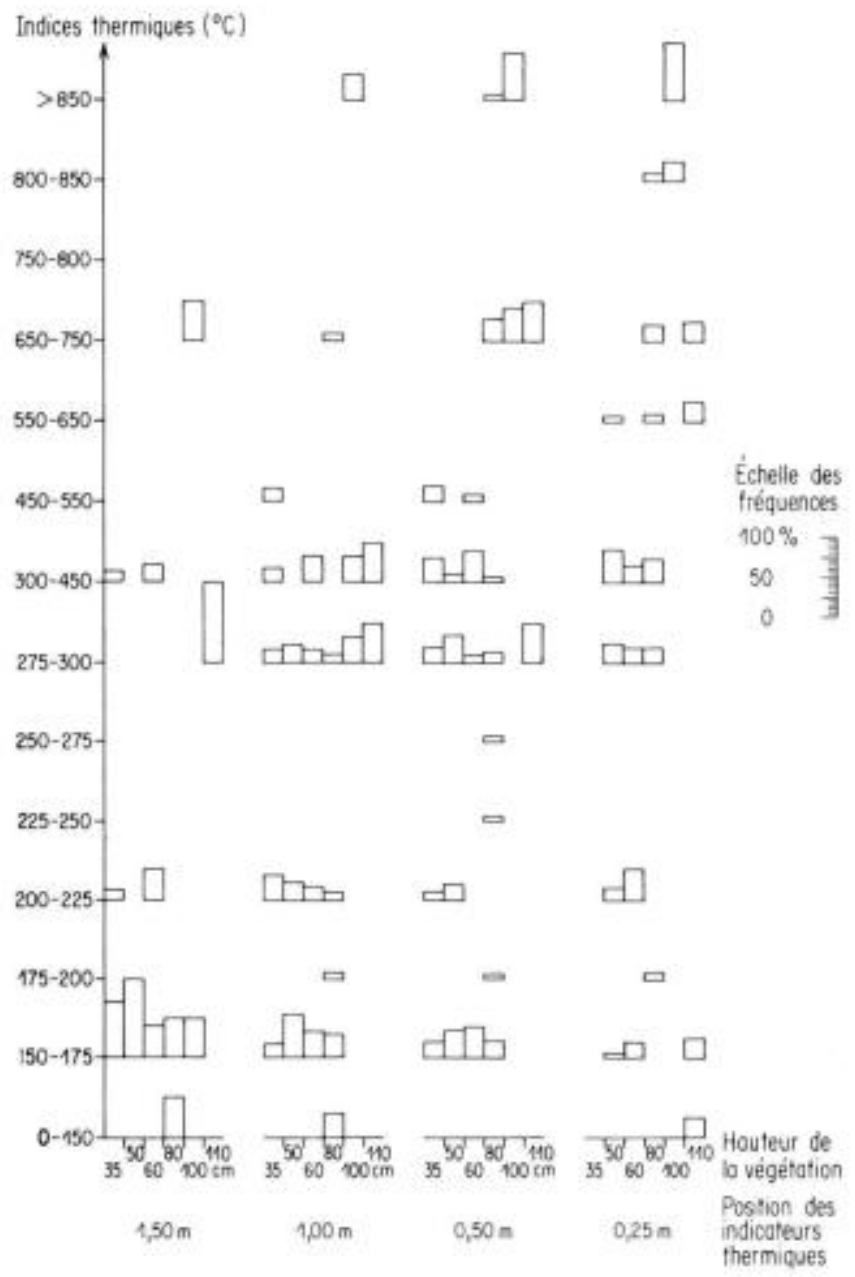

FIG. 6. - Fréquence (en p. 100) des indices thermiques en fonction de la hauteur de la végétation et de la position des indicateurs thermiques au-dessus de la surface du sol (feu de printemps).

Frequency (in p. 100) of the thermal indices according to the height of the vegetation and the location of the thermal indicators above soil surfoce (spring fires).

3.132. Températures observées dans le sol.

Les températures observées dans le sol sont peu élevées et diminuent très rapidement en fonction de la profondeur. La chaleur dégagée par la combustion des végétaux a peu d'effet sur les dispositifs de mesure (thermocouples et vernis sensibles) enterrés dans le sol. Les températures de $150^{\circ} \mathrm{C}$ n'apparaissent que rarement au-delà du premier centimètre ; en dessous de $5 \mathrm{~cm}$ de profondeur nous n'avons observé que des températures moyennes de $40^{\circ} \mathrm{C}$; à moins $10 \mathrm{~cm}$ il n'y a aucune élévation de température. 


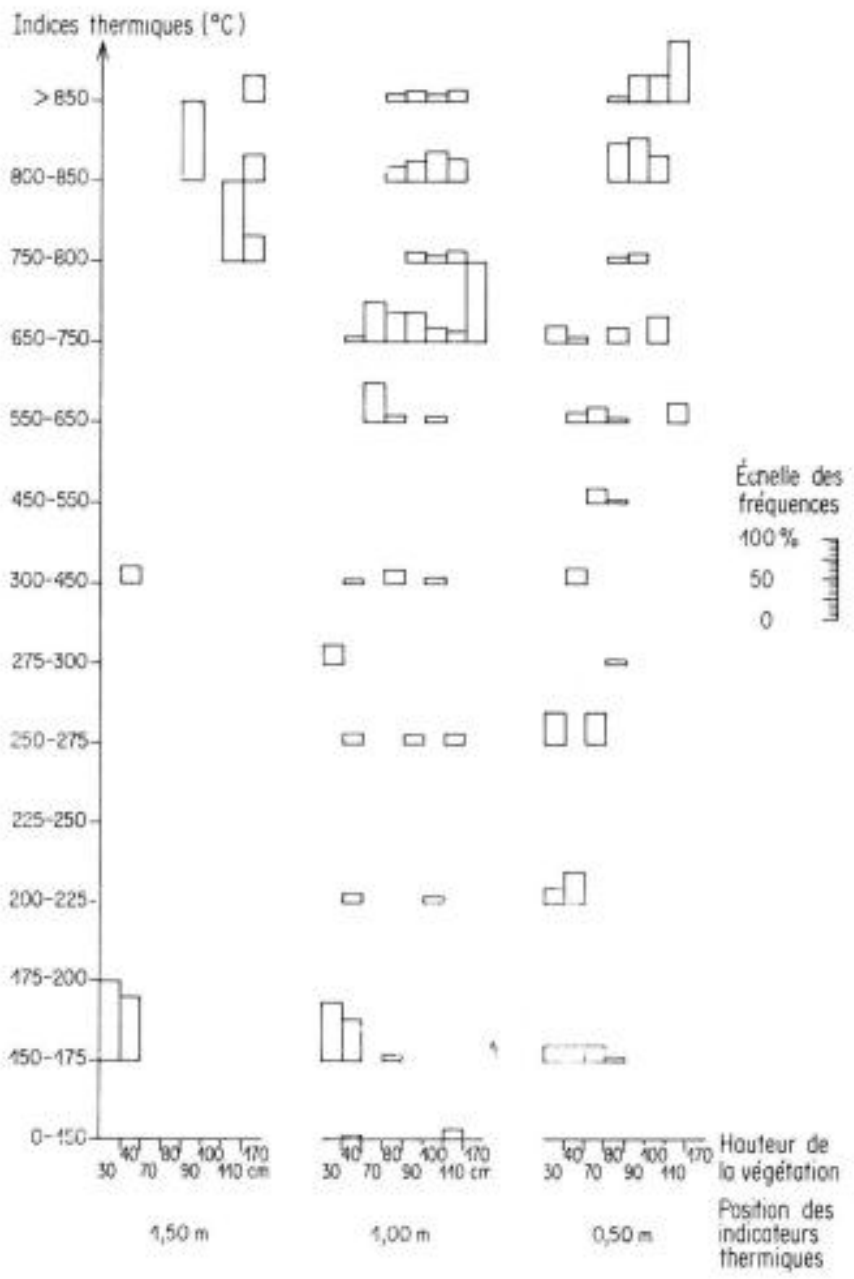

FIG. 7. - Fréquence (en p. 100) des indices thermiques en fonction de la hauteur de la végétation et de la position des indicateurs thermiques au-dessus de la surface du sol (feux d'automne).

Frequency (in p. 100) of the thermal indices according to the height of the vegetation and the location of the thermal indicators above soil surfoce (autumn fires).

La figure 8 représente la répartition des températures observées dans le sol. Les feux d'automne ont des températures toujours plus élevées que ceux de printemps, sauf dans la végétation déjà brôlée oủ il n'y a aucune différence.

Compte-tenu de nos résultats (deux cent cinq plaquettes avec vernis enterrées au cours de quarante et un brôlages), le flux thermique dans le sol obéit à la loi de diffusion des températures par conduction (Weil, 1965) :

$$
D \frac{\partial^{2} T}{\partial e^{2}}=\frac{\partial T}{\partial t}
$$



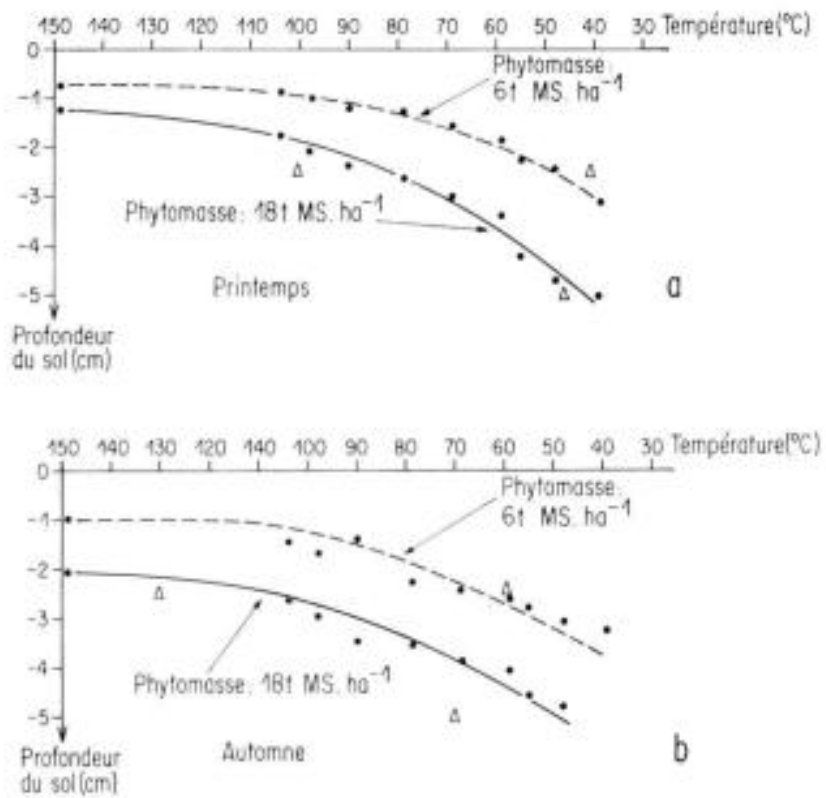

FIG. 8. - Répartition des températures observées dans le sol (répartition obtenue à partir des indicateurs thermiques, complétée par les mesures des thermocouples $\Delta$ ).

Distribution of the observed temperafures in the soil (distribution given by the thermal indicators, and the thermocouples data $\Delta$ ).

où $\mathrm{D}$ est le coefficient de diffusivité (pour la terre il est égal à $0,001 \mathrm{~cm}^{2} \cdot \mathrm{s}^{-1}$ ),

T est la différence de température,

$t$ est le temps,

e est l'épaisseur de la couche de terre considérée.

\subsection{Structure de la flamme et dispersion des températures.}

3.141. Les températures dans le sol et à la surface du sol.

Dans le sol, les températures de $150^{\circ} \mathrm{C}$ ne se manifestent que rarement au-delà du premier centimètre ; en outre, elles ne persistent qu'une à $2 \mathrm{mn}$ environ. Par contre, les températures de $50^{\circ} \mathrm{C}$ apparaissent au-delà de $5 \mathrm{~cm}$ de profondeur ef peuvent persister pendant plus de $30 \mathrm{mn}$.

A la surface du sol, les températures sont encore faibles, de l'ordre de 250 à $300^{\circ} \mathrm{C}$. Ce phénomène a d'ailleurs été observé par Vareschi (1962) dans la savane de Trachypogon au Vénézuéla, et Pitot et Masson (1971) au Sénégal.

\subsection{Les températures de la zone des combustibles végétaux.}

La température maximale, même quand le combustible végétal possède plusieurs strates, ne semble pas varier en fonction du nombre des strates. Lors de nos feux expérimentaux, les températures maximales atteignaient $1200^{\circ} \mathrm{C}$ dans la couche du combustible. Or, Countryman (1969), en Californie, constate que, quoique les strates soient plus ou moins nombreuses ou plus ou moins denses les températures maximales 
atteintes vont de 1040 à $1370^{\circ} \mathrm{C}$, même si la porosité des strates combustibles est assez faible pour restreindre fortement l'écoulement centripète de l'air vers l'intérieur des strates combustibles.

La porosité des strates combustibles augmente au fur et à mesure que le combustible est consumé, permettant ainsi à l'air ambiant de pénétrer plus amplement à l'intérieur de la couche combustible. Dès que la structure des strates combustibles se disjoint, il y a abaissement de la température maximale.

La figure 9 représente, de façon globale, la dispersion des températures à un même moment. Dans la couche combustible, à l'avant des flammes, progresse une zone de préchauffage qui s'accompagne de la pyrolyse des éléments chimiques des végétaux. Vient ensuite la zone de distillation des végétaux et de la combustion vive des distillats. C'est la zone des températures maximales. A l'arrière du front de flammes, les températures sont plus faibles, il n'y a plus qu'une combustion lente (le feu couve) amenant la calcination des débris végétaux.

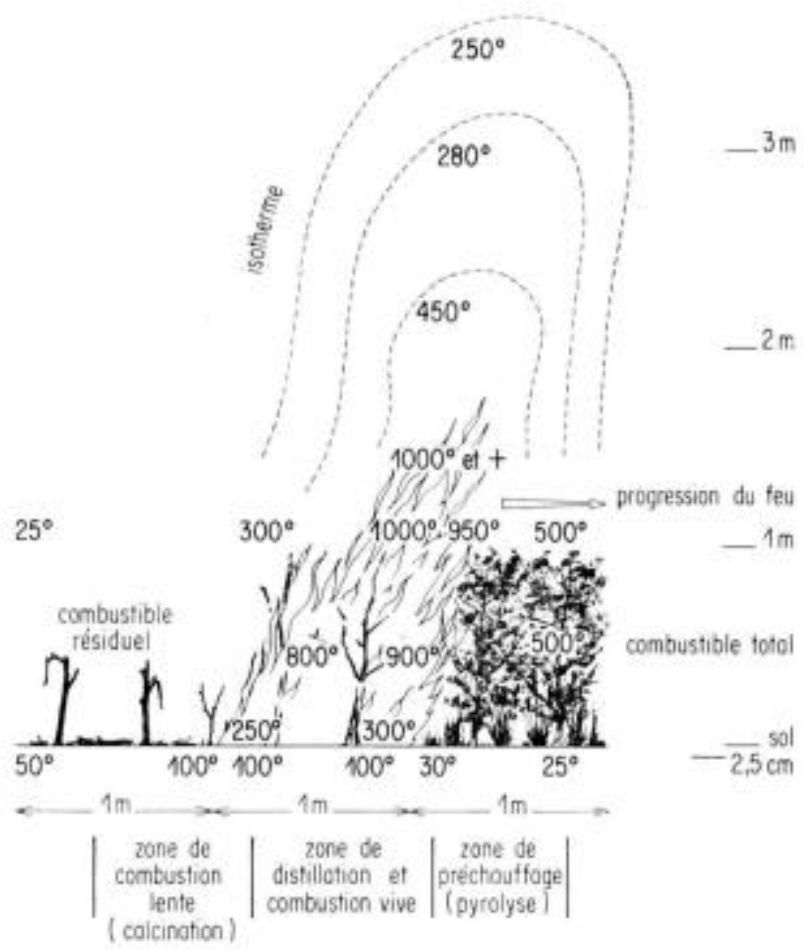

FIG. 9. - Structure du front de flammes et schéma de répartition des températures selon ies stades de la combustion.

Flame front structure and schema of the temperatures distribution according to the combustion sloges.

La température de l'air ambiant des combustibles en dehors de la zone en feu est rapidement tempérée par le flux d'air frais, ce qui fait que le préchauffage par rayonnement du combustible situé au devant d'un feu est un phénomène relativement lent. 


\subsection{Températures de la zone des flammes.}

De larges et rapides fluctuations caractérisent fortement les températures des flammes au-dessus du combustibles en train de brûler (fig. 9). L'écoulement turbulent de l'air qui mélange l'air ambiant plus frais aux flammes et aux gaz au-dessus de la couche combustible, et la pulsation, ou « tremblotement» des flammes, créent probablement la plus grande partie de cette fluctuation.

Les températures maximales dans la zone de combustion varient grandement et semblent être liées en partie à la vitesse de combustion. Certaines flammes sont massives et extrêmement turbulentes. Des fourbillons circulaires, composés entièrement de flammes, se forment souvent au-dessus du foyer en train de brûler.

3.144. Les températures des goz et de lo colonne de convection.

Au-dessus de la zone de combustion, les températures varient amplement en fonction du temps et de la hauteur (tabl. 2 et fig. 9 et 10).

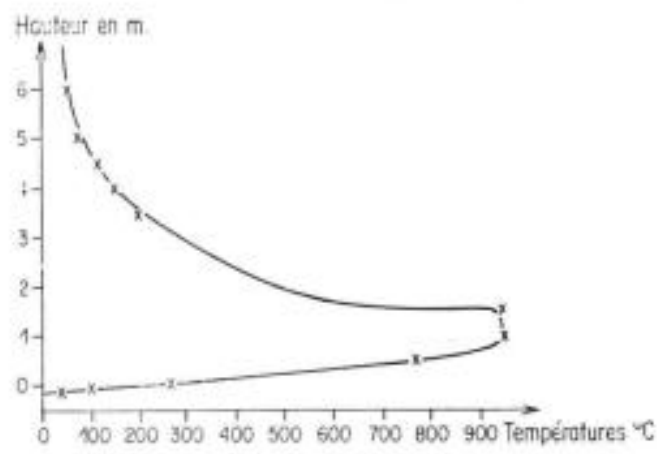

FIG. 10, - Dispersion des températures dans l'ensemble du milieu ambiant du feu (garrigue de Quercus coccifera L.).

Temperatures dispersion in fire environment (Quercus coccifera L. garrigue).

Au-dessus de la zone de combustion, les températures décroissent rapidement selon la hauteur. Les températures à l'intérieur du système en feu sont marquées par de larges variations spatiales et temporelles. Ces variations semblent dues à l'entraînement de l'air ambiant et à la forte furbulence à l'intérieur de la zone en feu. Autour des flammes, les gradients de tempérctures sont extrêmement brutaux. Au-dessus des flammes, dans la colonne de convection apparait un abaissement graduel des températures donnant des « isothermes» concentriques de plus en plus éloignées les unes des autres (fig. 9). Le long des bords de la colonne, il y a un refroidissement dû à la perte par rayonnement et à la turbulence.

Dans le sens latéral les températures décroissent très rapidement dès qu'on s'éloignent des flammes. Cette diminution de la température obéit aux lois de perte de la chaleur par rayonnement qui tiennent compte de la distance de l'objet soumis au rayonnement des flammes, de l'intensité du feu et de la conductivité de l'air.

Lors des brûlages de parcelles proches du poste météorologique, nous avons observé une élévation de la température, ainsi qu'un abaissement du taux de l'humidité relative de l'air, des appareils enregistreurs du poste (tabl. 3, fig. 11). 

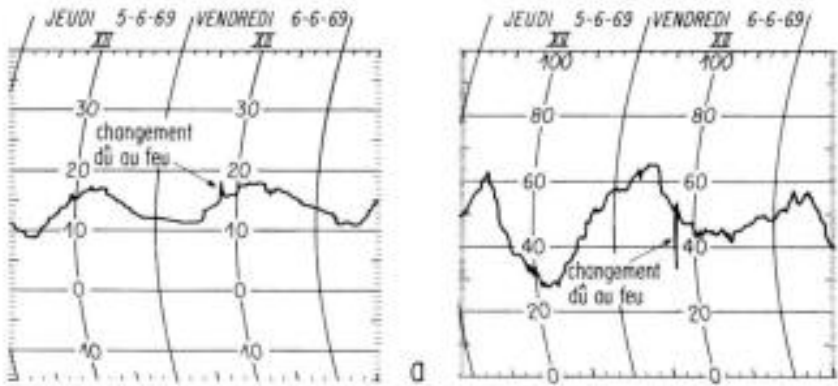

Temperoture

Humidité relative

Feu expérimentol du 6-6-69. Vent de NW, $5 \mathrm{~m} \cdot \mathrm{s}^{-1}$, distance $10 \mathrm{~m}$.

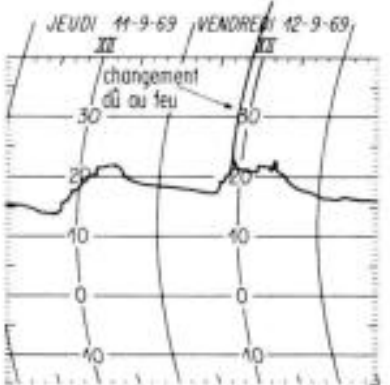

Temperoture

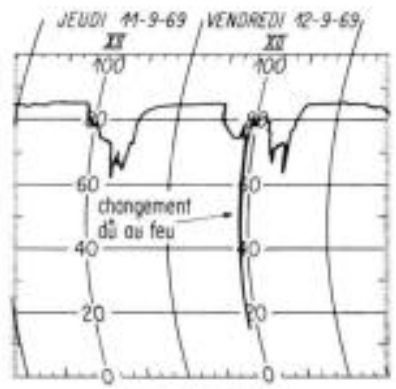

Humidté relative

Feu experimental du 12-9-69. Vent de $4 \mathrm{~m} .5^{-1}$, distonce $5 \mathrm{~m}$.

FIG. 11. - Exemples de changements de la température ef de l'humidité relative de l'air dus à la chaleur dégagée par le feu.

Examples of modifications in the air femperature and relative humidity due to the emitted heat from fire.

\section{TABLEAU 3}

Changements de la température ef de l'humidité relative de l'air au cours des feux (Modifications in the air temperatures and relative humidities during some fires)

\begin{tabular}{|c|c|c|c|c|c|c|c|}
\hline \multirow{2}{*}{ Date } & \multicolumn{2}{|c|}{ Température o $\mathrm{C}$} & \multicolumn{2}{|c|}{$\begin{array}{c}\text { Humidité relative } \\
\text { p. } 100\end{array}$} & \multicolumn{2}{|c|}{ Vent } & \multirow{2}{*}{$\begin{array}{l}\text { Distance de la } \\
\text { parcelle au poste } \\
\text { (m) }\end{array}$} \\
\hline & $\begin{array}{l}\text { avant } \\
\text { le feu }\end{array}$ & $\begin{array}{l}\text { pendant } \\
\text { le feu }\end{array}$ & $\begin{array}{l}\text { avant } \\
\text { le feu }\end{array}$ & $\begin{array}{l}\text { pendant } \\
\text { le feu }\end{array}$ & direction & $\begin{array}{l}\text { vitesse } \\
\mathrm{m} \cdot \mathrm{s}^{-1}\end{array}$ & \\
\hline $6 . \mathrm{VI} .69$ & 14,5 & 18,5 & 51 & 33 & NW & 4 & 10 \\
\hline $12.1 \times .69$ & 20,5 & 50 & 75 & 15 & SE & 4 & 5 \\
\hline $25.1 \times .75$ & 18,5 & 23 & 85 & 67 & S.SSE & 1 & 5 \\
\hline $25.1 \times .75$ & 18,5 & 20 & 86 & 81 & S-SSE & 1 & 10 \\
\hline $26.1 \times .75$ & 21,5 & 23,5 & 48 & 43 & NNW & 3 & $20-25$ \\
\hline
\end{tabular}


Ce qu'il faut noter n'est pas la température, ni l'humidité relative, absolve atteinte, mais plutôt la différence $\epsilon$ ntre les observations faites avant la mise à feu et celles faites pendant le feu. Ces effets sont dus à la vague de chaleur amenée par la colonne de convection poussée plus ou moins par le vent.

L'effet raffraîchissant de l'écoulement de l'air ambiant et convectif est probablement responsable des faibles températures mesurées ainsi que de la diminution considérable du rayonnement.

\section{2. - Les vitesses de propagation du feu}

Le phénomène de la propagation du feu est complexe. Ce sont les mécanismes les plus simples de la combustion (rayonnement, convection, conduction) qui commandent la propagation du feu, mais aussi d'autres éléments tels que : la vitesse du vent, la phytomasse disponible, la teneur en eau ef la porosité de la couche combustible, la pente du terrain.

\subsection{Revue de la documentation.}

A notre connaissance, peu d'auteurs ont publié des résultats sur les vitesses de propcgation. Byram (1959) dit qu'un feu en train de brôler dans une tourbière ne progresse que de quelques mètres en plusieurs semaines ; un feu «prescrit » peut parcourir en moyenne $0,9 \mathrm{~d} 1,3 \mathrm{~cm} \cdot \mathrm{s}^{-1}$; par contre, un feu sauvage peut se propager à une vitesse otteignant 139 à $167 \mathrm{~cm} \cdot \mathrm{s}^{-1}\left(5\right.$ à $\left.6 \mathrm{~km} \cdot \mathrm{h}^{-1}\right)$. Mc Arthur $(1960,1966)$ trouve que, selon la vitesse du vent ef la teneur en eau des végétaux, les feux de Graminées, en Australie, peuvent varier entre $8 \mathrm{~cm} \cdot \mathrm{s}^{-1}$ et $350 \mathrm{~cm} \cdot \mathrm{s}^{-1}\left(12,8 \mathrm{~km} \cdot \mathrm{h}^{-1}\right)$. De même, Mc Arthur (1967) donne quelques indications sur les vitesses de propagation dans les forêts d'Eucalyptus ; ces vitesses peuvent aller de $1 \mathrm{~cm} \cdot \mathrm{s}^{-1}$ à $84 \mathrm{~cm} \cdot \mathrm{s}^{-1}$ en fonction de la vitesse du vent, de l'humidité relative et de la phytomasse. Au Canada, Van Wagner $(1965 a, 1965 b, 1966)$ constate des vitesses de propagation allant de $2,5 \mathrm{~cm} .5^{-1}$, pour les brôleges «prescrits » de déchets d'abattage, jusqu'à $40 \mathrm{~cm} \cdot \mathrm{s}^{-1}$ pour des feux de cimes.

Lors des feux expérimentaux en milieu de garrigue, nous avons observé les diverses vitesses de propagation en foncticn des trois paramètres que nous pouvions connaître : la vitesse du ver.t, la havieur de la végétation, qui peut être un bon indice de la quantité de matériel végétal ccmbustible, $\epsilon t$ la teneur en eau.

La pente du terroin étant toujours la même, nous ne l'avons pas prise en considération. II ne faut pas oublier aussi que, compte tenu de la petite taille des parcelles, les résultats obtenus représentent, peut être, des valeurs inférieures à celles qui auraient pu être observées dans de grands feux.

\subsection{Effets des différents paramètres considérés sur la vitesse de propagation.}

3.221. La vitesse du vent influence la vitesse de propagation du feu. Cela est bien connu ; plus un vent sera fort, plus le feu ira vite.

La figure 12 donne les vitesses de propagation du feu en fonction de la vitesse du vent et de la hauteur de la végétation qui sont, lors de nos expérimentations les deux paramètres qui influencent le plus cette propagation. 


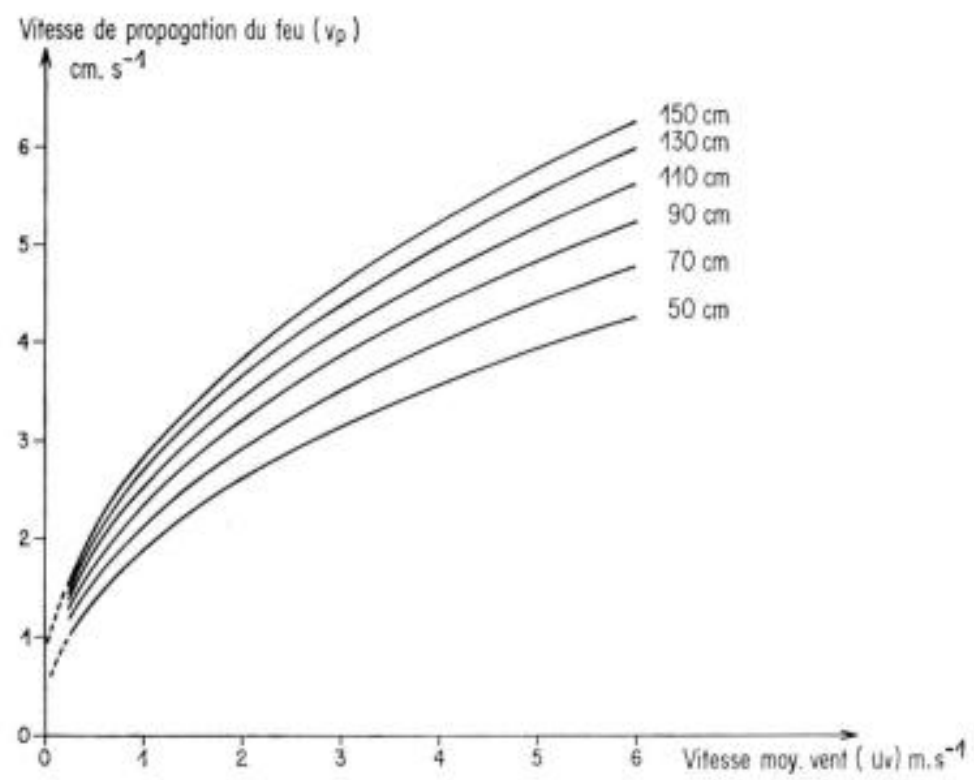

FIG. 12. - Vilesse de propagation du feu en fonction de la vitesse moyenne du vent et de differentes hauteurs du toit de la végétation (garrigue de Quercus coccifera L.).

Fire rate of spread according to the mean wind speed and different heights of the vegetation (Quercus coccifera L. garrigue).

L'équation obtenue est égale à :

$$
\mathrm{vp}=0,066 \mathrm{Uv}^{0,439} \mathrm{H}^{0,315} \quad \mathrm{r}=0,84
$$

où $v p=$ vitesse de propagation du feu en $\mathrm{cm} \cdot \mathrm{s}^{-1}$,

$\mathrm{Uv}=$ vitesse moyenne du vent en $\mathrm{cm} \cdot \mathrm{s}^{-1}$,

$\mathrm{H}=$ hauteur de la végétation en $\mathrm{cm}$.

3.222. Il en est de même pour la hauteur de la végétation ; la phytomasse aérienne (traduite ici par la hauteur) doit contribuer à l'accélération de la vitesse de propagation du feu jusqu'à un certain point, au-delà duquel il n'y a plus accroissement de la vitesse. Mc Arthur (1967) pense que, dans les grands incendies, quand le feu a atteint un stade d'équilibre, la propagation est indépendante des autres facteurs.

3.223. La teneur en eau du combustible a aussi un effet sur la vitesse de propagation, mais à un moindre degré. Elle a tendance à ralentir la vitesse de propagation ; c'est-àdire, plus la teneur en eau du combustible est élevée plus la vitesse de propagation est réduite. En considérant la hauteur de la végétation constante, plus un combustible est sec, ef plus le vent est fort, plus la vitesse de propagation sera grande.

Si nous introduisons cette troisième variable nous obtenons l'équation suivante :

$$
v p=\frac{5,72 \cdot U v^{0,400} H^{0,352}}{t e^{1,12}} \quad r=0,88
$$

où te est la teneur en eau, en pourcentage du poids de matière verte. 


\section{3. - Hauteurs des flammes}

Au cours du feu ; la hauteur de la flamme est appréciée visuellement pour l'ensemble de la parcelle considérée.

La hauteur de la flamme est directement liée à la vitesse de propagation du feu et à la quantité de combustible. La relation entre la vitesse de propagation, la hauteur de la végétation et la hauteur de la flamme est une courbe dont l'équation est :

$$
\text { If }=12,33 \times p^{0,428} \mathrm{H}^{0,477} \quad \mathrm{r}=0,83
$$

oủ If = hauteur de la flamme en $\mathrm{cm}$.

Plus la vitesse de propagation est rapide plus la hauteur de la flamme est grande (fig. 13).

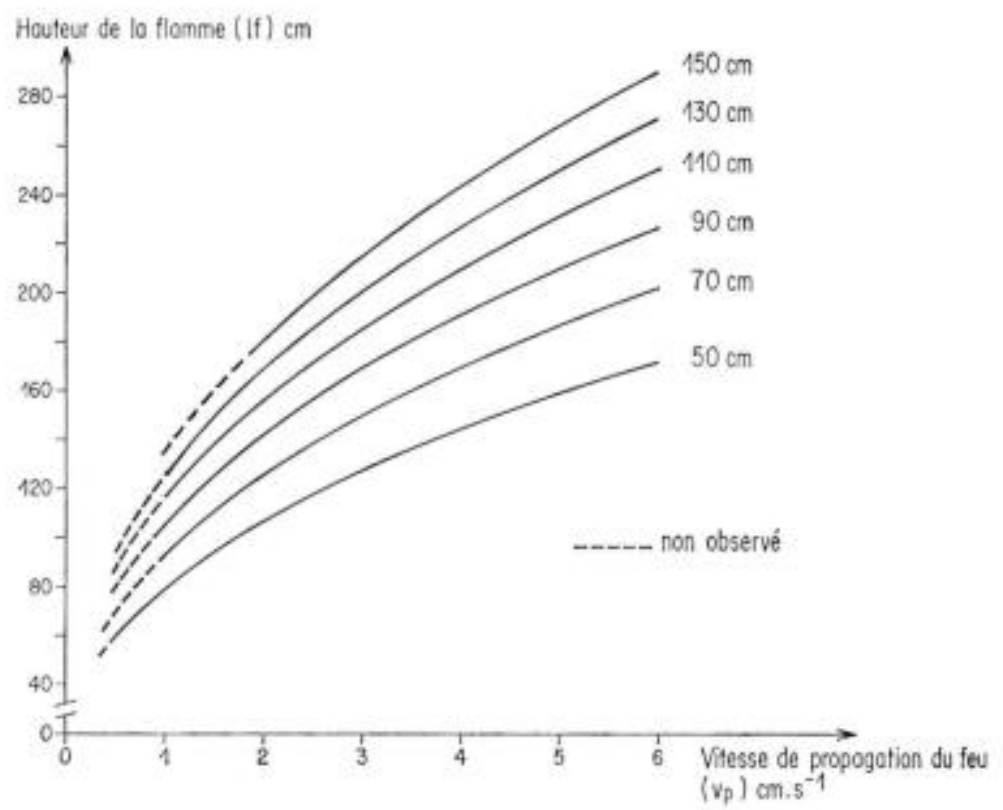

FIG. 13. - Hauteur de la flamme en fonction de la vitesse de propagation du feu et de différentes hauteurs du toir de la végétation (garrigue de Quercus coccifera L.).

Flame height according to the fire rate of spread and different heights of the vegetation (Quercus coccifera L. garrigue).

Néanmoins cette hauteur ne croîtra pas indéfiniment.

Mc Arthur (1967) déclare que les hauteurs maximales des flammes apparaissent quand la vitesse moyenne du vent oscille entre 30 et $40 \mathrm{~km}$ à l'heure. Bien qu'elles aient été faites dans les forêts d'Eucalyptus, ces observations sont semblables aux nôtres oủ, apparemment, les hauteurs maximales des flammes seraient atteintes pour des vitesses du vent de l'ordre de $10 \mathrm{~m} \cdot \mathrm{s}^{-1}$ (36 km à l'heure).

La hauteur de la végétation n'étant qu'un indice de la phytomasse aérienne, il est aussi raisonnable de penser que la quantité et la distribution du combustible jouent 
un rôle primordial en déterminant la hauteur de la flamme ; les hauteurs des flammes s'accroissent notablement au fur ef à mesure qu'il y a de plus en plus de combustible disponible.

\section{4. - Energie dégagée par les feux}

L'énergie dégagée par un feu peut être calculée lorsque plusieurs paramètres sont connus. Cette énergie correspond à la « puissance du front de feu » Pf (Byram, 1959 ; Tangren, 1976) qui est liée à la phytomasse en train de brûler et à la vitesse de propagation du feu ; elle mesure la quantité de chaleur dégagée par unité de temps et de longueur de front de feu. Comme il faut un certain laps de temps pour que le combustible végétal soit brûlé, la chaleur dégagée ne sera pas confinée à la partie avant du feu, mais s'étendra sur toute la largeur de la bande qui est en combustion.

Byram (1959) et Tangren (1976) ont donné l'équation qui permet de calculer la «puissance linéaire du front du feu » : $\mathrm{Pf}=\mathrm{Hwr}$,

oủ $\mathrm{Pf}=$ puissance du front du feu en calories par seconde et par centimètre.

$\mathrm{H}=$ chaleur de combustion (ou valeur énergétique), en calories par gramme de combustible :

$w=$ masse de combustible effectivement brûlé, en grammes par centimètre carré ;

$r=$ vitesse de propagation du feu, en centimètres par seconde.

Cette équation permet de caractériser les feux. C'est une valeur globale qui comprend l'énergie dissipée par convection, rayonnement et conduction.

La chaleur de combustion (ou valeur énergétique) varie selon les différents végétaux et selon les organes des végétaux. La masse de combustible effectivement brôlé correspond à la différence entre la phytomasse avant brôlage ef la phytomasse restant après brôlage. Quant à la vitesse de propagation, elle peut varier considérablement entre les différents feux.

Ainsi, si l'on dispose des données adéquates sur la masse du combustible disponible, la puissance du front de feu peut être calculée après avoir mesuré seulement la vitesse de propagation.

Afin de connaître quelle était la proportion de matériel végétal brôlé au cours des feux, nous avons brûlé trois parcelles hors expérimentation. La phytomasse moyenne de matière sèche était de 22,6 † à l'ha avant feu ; après le feu 10,1 t de matière sèche restent en place, soit une phytomasse de 12,5 t brôlée au cours des feux. Ce qui donne $0,125 \mathrm{~g}$ de matière sèche par $\mathrm{cm}^{2}$ consumé.

La chaleur de combustion a été prise comme $19,5 \mathrm{~kJ} / \mathrm{g}$ de matière sèche, valeur énergéfique avancée par Heim (1974) que nous avons considérée comme valeur moyenne, compte tenu du combustible qui brôle. Cela donne $4658,9 \mathrm{cal} / \mathrm{g}$ de matière sèche.

En remplaçant les symboles de l'équation par ces différentes valeurs, nous pouvons alors calculer la puissance du front de feu. Celle-ci serait située entre $1164,7 \mathrm{cal}$. $\mathrm{cm}^{-1} \cdot \mathrm{s}^{-1}$ (pour une vitesse de propagation de $2 \mathrm{~cm} \cdot \mathrm{s}^{-1}$ ) et $3494,2 \mathrm{cal} \cdot \mathrm{cm}^{-1} \cdot \mathrm{s}^{-1}$ (pour une vitesse de propagation de $6 \mathrm{~cm} \cdot \mathrm{s}^{-1}$ ), pour une garrigue typique de Chêne kermès d'un mètre de haut. 
Dans les parcelles oủ la végétation avait déjà brûlée plusieurs fois (phytomasse comprise entre 4 et $6 \mathrm{t} . \mathrm{ha}^{-1}$ ) constituée principalement par Brachypodium ramosum, la puissance du front de feu oscillait entre 153 et $1152 \mathrm{cal} . \mathrm{cm}^{-1}, \mathrm{~s}^{-1}$, selon la vitesse de propagation (respectivement $0,6 \mathrm{~cm} \cdot \mathrm{s}^{-1}$ et $2,2 \mathrm{~cm} \cdot \mathrm{s}^{-1}$ ).

La puissance de nos feux est donc relativement faible puisque selon Byram (1959) la puissance peut aller de $40 \mathrm{cal} \cdot \mathrm{cm}^{-1} \cdot \mathrm{s}^{-1}$ pour des feux légers de graminées se propageant contre le feu, jusqu'à $250000 \mathrm{cal} . \mathrm{cm}^{-1}$. $\mathrm{s}^{-1}$ lors des grands feux de forêts brûlant la cîme des arbres et se propageant à de grandes vitesses. Comparées avec les données de Van Wagner $(1965 a, 1965 b, 1966)$, au Canada, nos données correspondent à un feu de sous-bois de forêt de Pinus resinosa ou à un feu de déchets d'abattage, se propageant relativement lentement. Par contre, les grands feux rapides brûlant des déchets d'abattage sont plus intenses. Les feux les plus intenses, évidemment sont ceux qui consument le maximum de combustible, c'est-à-dire les feux qui brûlent à la fois le sous-bois et le feuillage des arbres, leur puissance atteint alors $50000 \mathrm{cal} \mathrm{cm}^{-1} \cdot \mathrm{s}^{-1}$ environ.

\section{4. - Conclusion}

Ces expériences inédites sur le « comportement du feu » dans la garrigue de Chêne Kermès apportent des précisions sur le phénomène des feux de végétation dans le midi méditerranéen. Quoique réalisées sur des parcelles expérimentales, les données présentées correspondent très bien au comportement des feux sauvages dans ce type de végétation. En effet, elles sont semblables aux résultats obtenus par d'autres chercheurs ailleurs dans le monde. Ce qui permet de supposer une grande convergence du comportement du feu quel que soit le peuplement végétal qui brûle.

Par ailleurs, l'application à la lutte anti-incendie de tels résultats peut être importante. En effet, l'ensemble des informations fournies caractérise le comportement du feu compte tenu de la structure du peuplement végétal et des conditions météorologiques dominantes au moment du feu. Ceci devrait permettre au combattant du feu de connaitre très rapidement la situation d'un incendie dans ce type de peuplement; et donc de décider très rapidement du choix des moyens à mettre en œuvre en hommes et en matériel, pour lutter et maîtriser plus efficacement et plus rapidement le feu.

Reçu pour publication en juillet 1978.

\section{Remerciements}

Nous remercions tous les nombreux techniciens et ouvriers qui nous ont aidé lors des mises à feu. Nous remercions aussi $M$. Rambal qui nous a conseillé lors de l'interprétation mathématique des résultats. Enfin nous ne saurions oublier $M$. Long qui nous a constamment guidé lors de ce travail et qui a bien voulu revoir le texte du manuscrit.

\section{Summary}

Study of fire behavior in a Quercus coccifera L. garrigue

Fire behavior may be characterized in different ways. Temperature, rate of spread, height of the flames and fire energy can be used as expressions of this behavior. Temperatures fluctuate very 
rapidly ; moreover, they differ according to the location of the probes (in the ground, in the vegetation. above the vegetation) and according to the time of burning (spring or autumn). The highest temperatures are always observed near the top of the vegetation and during autumn burnings. From the results, an attempt is made to represent flame structure and dispersion of temperatures in the flame front. The rate of spread closely depends on wind speed and height of the vegetation. Flame height is correlated with the rate of spread of the fire and height of the vegetation. The energy emitted by fires is measured by means of the « fire front power $»$. Energies recorded during the experimental burnings are low.

The results allow to better understand fire behavior in a Quercus coccifera L. garrigue. They are also of great importance for fire fighting operations.

\section{Références bibliographiques}

BARROWS J. S., 1951. Fire behaviour in the Northern Rocky Mountains forests. U. S. Forest Serv., Northern Rocky Mountain Forest and Range Exp. Sta., Sta. Paper no 29, 103 p.

BYRAM G. M., 1959. Combustion of forest fuels, in Forest Fire : Control and Use. K. P. Davis Ed. Me Graw-Hill Book Co New York : 61-89.

COUNTRYMAN C. M., 1964. Mass fires and fire behoviour. U. S. Forest Serv., Pacific Southwest Forest and Range Exp. Sta., Res. Pap. PSW-19, 53 p.

COUNTRYMAN C. M., 1969. Project Flombeau an investigation of mass fire (1964-1967) Final report. Pacific Southwest Forest and Range Exp. Sta., 68 p.

EMBERGER L., 1942. Un projet de classification des climats du point de vue phyłogéographique. Bull. Soc. hist. nat. Toulouse, $77: 97-124$.

EMBERGER L., 1971. Considérations complémentaires au sujet des recherches bioclimatologiques et phytogéographiques-écologiques, in Travaux de Botanique et d'Ecologie. Masson et Cie, Paris, 291-301.

FENNER R. L., BENTLEY J. R., 1960. A simple pyrometer for measuring soil temperatures during wildland fires. Pacific Southwest Forest and Range Exp. Sta., Misc. Pap. no 45, 9 p.

HEIM G., 1974. L'utilité du concept de valeur énergétique en écologie. Une étude basée sur des mesures effectuées sur des plantes méditerranéennes. Oecol. Plant., $9: 281-286$.

IWANAMI Y., 1973. Studies on burning temperatures of grasslands. Rep. Inst. Agr. Res. Tohoku Univ, $24: 59-105$.

KAYLL A. J., 1966. Some characteristics of heath fires in northeast Scotland. J. Appl. Ecology, 3 : 29-40.

LONG G., VISONA L., RAMI J., 1961. La végétation du domaine de Coulondres (Hérault). Relation avec les problèmes de mise en valeur. Boll. Inst. Bot. Univ. Catonia, 3 : 5-52.

LONG G., FAY F., THIAULT M., TRABAUD L., 1967. Evolution de la masse végétale en milieu de garrigue soumise à diverses inferventions humaines. CEPE. Montpellier. Doc. $n^{\circ} 39,28 \mathrm{p}$.

Mc ARTHUR A. G., 1960. Fire danger rating tables for annual grosslands. Aust. Forest. and Timber Bur., 15 p.

Mc ARTHUR A. G., 1966. Weather and grassland fire behaviour. Aust. Forest. and Timber Bur., $n^{0} 100$, $23 \mathrm{p}$.

Mc ARTHUR A. G., 1967. Fire behaviour in Eucalypt forests. Aust. Forest. and Timber Bur., leaflet $n^{\circ} 107,36 \mathrm{p}$.

MC ARTHUR A. G., CHENEY N. P., 1970. The fire behaviour characteristics of the Bossin Creek Fire. 29th December 1967. 1 st January 1968. Forest Res. Inst., Forest. and Timber Bur., 33 p.

MARTIN R. E., CUSHWA C. T., MILLER R. L., 1969. Fire as a physical factor in wildland management. Proc. Ann. Tall Timbers Fire Ecol. Conf., 9 : 271-288.

PITOT A., MASSON H., 1951. Quelques données sur la température au cours des feux de brousse aux environs de Dakar. Bull. Inst. Afr. Noire, 13 : 711-732.

POISSONET P., 1966. Etude méthodologique en écologie végétale da partir des photographies aériennes. Thèse de $3^{\mathrm{e}}$ cycle (Ecologie) Fac. Sci. Montpellier, $107 \mathrm{p}$.

SMITH D. W.. SPARLING J. H., 1966. The temperatures of surface fire in jack pine barren, 1 et 11. Can. J. Bot., $44: 1285-1298$.

STINSON K. J., WRIGHT M. A., 1969. Temperatures of head fires in the southern mixed prairies of Texas. J. Range Manag., $22: 169-174$.

TANGREN C. D., 1967. The trouble with fire intensity. Fire Techn., $12: 261-265$.

TRABAUD L., 1962. Monogrophie phytosociologique et écologique de la région de Gratels. St. Gély du Fesc. Thèse de $3^{\circ}$ cycle (Ecologie) Fac. Sci. Montpellier, $131 \mathrm{p}$.

TRABAUD L., 1974. Experimental study on the effects of prescribed burning on a Quercus coccifera L. garrigue, Early results. Proc. Ann. Tall Timbers Fire Ecol. Conf., 13 : 97-129.

TRABAUD L., 1975. Les températures des feux de végétation. Deux. Symp. Europ. Combustion. Orléans, 1.5 septembre $1975,1: 210-214$. 
TRABAUD L., 1976. Inflammabilité et combustibilité des principales espèces de.la garrigue. Oecol. Plant., $11: 117-136$.

TRABAUD L., 1977. Comparison between the effect of prescribed fires and wild fires on the global quantitative evolution or the Kermes scrub oak ( $Q$ coccifera L.) garrigues. Proc. Symp. Environ. Consequences of Fire and Fuel Manog. in Medit. Ecosystems, 217-282.

VARESCHI V., 1962. La quema como factor ecologico en los Llanos. Bol. Soc. Venezol. Cienc. Nat., $23: 9-26$.

WAGNER C. E. Van, 1965a. Story on an intense crown fire at Petawawa. Pulp and Pap. Mag. Canada $66: 358-361$.

WAGNER C. E. Van, 1965b. Describing forest fires - old ways and new. Forestry Chronicle, 41 : 301-305.

WAGNER C. E. Van, 1966. Three experimental fires in jack pine slash. Can. Forest. Serv., Depart. Forest. Pub. $\mathrm{n}^{\circ} 1146,22 \mathrm{p}$.

WEIL L., 1965, - Eléments des échanges thermiques. Gauthier-Villars. Paris, 226 p.

WHITTAKER E., 1961. - Temperatures in heath fires. J. Ecol., 49:709-714. 